\title{
Optimal bilinear control of nonlinear Schrödinger equations with singular potentials
}

\author{
Binhua Feng*, Dun Zhao, Pengyu Chen \\ School of Mathematics and Statistics, Lanzhou University \\ Lanzhou, 730000, P.R. China
}

\begin{abstract}
In this paper, we consider an optimal bilinear control problem for the nonlinear Schrödinger equations with singular potentials. We show well-posedness of the problem and existence of an optimal control. In addition, the first order optimality system is rigorously derived. Our results generalize the ones in [11] in several aspects.
\end{abstract}

Keywords: Optimal bilinear control; Nonlinear Schrödinger equation; Optimal condition

\section{Introduction}

This paper is devoted to study an optimal bilinear control problem for the following nonlinear Schrödinger equation(NLS):

$$
\left\{\begin{array}{l}
i u_{t}+\Delta u+\lambda|u|^{2 \sigma} u+\phi(t) V(x) u=0,(t, x) \in[0, \infty) \times \mathbb{R}^{N}, \\
u(0, x)=u_{0}(x),
\end{array}\right.
$$

where $u_{0} \in H^{1}\left(\mathbb{R}^{N}\right), \phi(t)$ denotes the control parameter and $V(x)$ is a given potential. The problem of quantum control via external potentials $\phi(t) V(x)$, has attracted a great deal of attention from physicians, see [1, 2, 3]. From the mathematical point of view, quantum control problems are a specific example of optimal control problems, see 4], which consist in minimizing a cost functional depending on the solution of a state equation (here, equation (1.1)) and to characterize the minimum of the functional by an optimality condition.

The mathematical research for optimal bilinear control of systems governed by partial differential equations has a long history, see [5, 6] for a general overview. However, there are only a few rigorous mathematical results about optimal bilinear control of Schrödinger equations. Recently, optimal control problems for linear Schrödinger equations have been investigated in [7, 8, 9]. Moreover, those results have been tested numerically in [9, 10]. In particular, a mathematical framework for optimal bilinear control of abstract linear Schrödinger equations was presented in [7]. In [8], the authors considered the optimal bilinear control for the linear

E-mail: binhuaf@163.com(Binhua Feng)

${ }^{*}$ Corresponding author

This work is supported by the Program for the Fundamental Research Funds for the Central Universities, NSFC Grants 11031003 and 11171028, and the Program for NCET. 
Schrödinger equations including coulombian and electric potentials. For the following NLS of Gross-Pitaevskii type:

$$
\left\{\begin{array}{l}
i u_{t}+\Delta u-U(x) u-\lambda|u|^{2 \sigma} u-\phi(t) V(x) u=0,(t, x) \in[0, \infty) \times \mathbb{R}^{N} \\
u(0, x)=u_{0}(x)
\end{array}\right.
$$

where $\lambda \geq 0, U(x)$ is a subquadratic potential, consequently restricting initial data $u_{0} \in \Sigma:=$ $\left\{u \in H^{1}\left(\mathbb{R}^{N}\right)\right.$, and $\left.x u \in L^{2}\left(\mathbb{R}^{N}\right)\right\}$. The authors in [1] have presented a novel choice for the cost term, which is based on the corresponding physical work performed throughout the control process. The proof of the existence of an optimal control relies heavily on the compact embedding $\Sigma \hookrightarrow L^{2}\left(\mathbb{R}^{N}\right)$. In contrast with (1.2), due to absence of $U(x) u$ in (1.1), we consider (1.1) in $H^{1}\left(\mathbb{R}^{N}\right)$. Therefore, how to overcome the difficulty that embedding $H^{1}\left(\mathbb{R}^{N}\right) \hookrightarrow L^{2}\left(\mathbb{R}^{N}\right)$ is not compact, which is of particular interest, is one of main technique challenges in this paper.

Borrowing the idea of [11, we now define our optimal control problem. The natural candidate for an energy corresponding to (1.1) is

$$
E(t)=\frac{1}{2} \int_{\mathbb{R}^{N}}|\nabla u(t, x)|^{2} d x-\frac{\lambda}{2 \sigma+2} \int_{\mathbb{R}^{N}}|u(t, x)|^{2 \sigma+2} d x-\frac{\phi(t)}{2} \int_{\mathbb{R}^{N}} V(x)|u(t, x)|^{2} d x .
$$

Although equation (1.1) enjoys mass conservation, i.e., $\|u(t, \cdot)\|_{L^{2}}=\left\|u_{0}\right\|_{L^{2}}$ for all $t \in \mathbb{R}$, the energy $E(t)$ is not conserved. Indeed, its evolution is given by

$$
\frac{d E(t)}{d t}=-\frac{1}{2} \phi^{\prime}(t) \int_{\mathbb{R}^{N}} V(x)|u(t, x)|^{2} d x .
$$

Integrating this equality over the compact interval $[0, T]$, we obtain

$$
E(T)-E(0)=\frac{1}{2} \int_{0}^{T} \phi^{\prime}(t) \int_{\mathbb{R}^{N}} V(x)|u(t, x)|^{2} d x d t .
$$

For any given $T>0$, we consider $H^{1}(0, T)$ as the real vector space of control parameters $\phi$. Set

$$
X(0, T):=L^{2}\left((0, T), H_{0}^{1}\right) \cap W^{1,2}\left((0, T), H^{-1}\right),
$$

. For some $M_{1}>0$ and $M_{2}>0$, set $B_{1}:=\left\{u_{0} \in H^{1}\right.$ and $\left.\left\|u_{0}\right\|_{H^{1}} \leq M_{1}\right\}$ and $B_{2}:=\left\{\phi_{0} \in\right.$ $\mathbb{R}$ and $\left.\left|\phi_{0}\right| \leq M_{2}\right\}$

$\Lambda(0, T):=\left\{(u, \phi) \in X(0, T) \times H^{1}(0, T): u\right.$ is the solution of (1.1) with $u(0) \in B_{1}$ and $\left.\phi(0) \in B_{2}\right\}$.

Thanks to Lemma 2.3, the set $\Lambda(0, T)$ is not empty. We consequently define the objective functional $F=F(u, \phi)$ on $\Lambda(0, T)$ by

$$
F(u, \phi):=\langle u(T, \cdot), A u(T, \cdot)\rangle_{L^{2}}^{2}+\gamma_{1} \int_{0}^{T}\left(E^{\prime}(t)\right)^{2} d t+\gamma_{2} \int_{0}^{T}\left(\phi^{\prime}(t)\right)^{2} d t,
$$

where parameters $\gamma_{1} \geq 0$ and $\gamma_{2}>0, A: H^{1} \rightarrow L^{2}$ is a bounded linear operator, essentially self-adjoint on $L^{2}$ and localizing, i.e., there exists $R>0$, such that for all $\psi \in H^{1}$ : $\operatorname{supp}_{x \in \mathbb{R}^{N}}(A \psi(x)) \subseteq B(R)$. 
Now, we can define the following minimizing problem:

$$
F_{*}=\inf _{(u, \phi) \in \Lambda(0, T)} F(u, \phi) .
$$

Firstly, we consider the existence of a minimizer for the above minimizing problem. This is what the following theorem shows:

Theorem 1.1. Assume $0<\sigma<\frac{2}{N-2}$ if $\lambda<0$, or $0<\sigma<\frac{2}{N}$ if $\lambda>0$. Let $V \in L^{p}+L^{\infty}$ for some $p \geq 1, p>N / 2$. Then, for any $T>0, M_{1}>0, M_{2}>0, \gamma_{1} \geq 0$ and $\gamma_{2}>0$, the optimal control problem (1.8) has a minimizer $\left(u_{*}, \phi_{*}\right) \in \Lambda(0, T)$.

Remarks. (1) In contrast with the results in [11], our results hold for unbounded potential $V$, both focusing and defocusing nonlinearities. A typical example satisfying our assumption on $V$ is $\frac{1}{|x|^{\alpha}}$ for some $0<\alpha<1$.

(2) Since the embedding $H^{1}\left(\mathbb{R}^{N}\right) \hookrightarrow L^{2}\left(\mathbb{R}^{N}\right)$ is not compact, the method in [11] fails to work in our situation. We can derive the compactness of a minimizing sequence by Propositions 1.1.2 and 1.3 .14 in [12].

Thanks to global well-posedness of equation (1.1), for any given initial data $u_{0} \in H^{1}$, we can define a mapping by

$$
u: H^{1}(0, T) \rightarrow X(0, T): \quad \phi \mapsto u(\phi) .
$$

Using this mapping we introduce the unconstrained functional

$$
\mathcal{F}: H^{1}(0, T) \rightarrow \mathbb{R}, \quad \phi \mapsto \mathcal{F}(\phi):=F(u(\phi), \phi)
$$

In the following theorem, we investigate the differentiability of unconstrained functional $\mathcal{F}$, and consequently obtain the first order optimality system.

Theorem 1.2. Let $N \leq 3, u_{0} \in H^{2}, V, \nabla V \in L^{p}+L^{\infty}$ and $V \in L^{2 p}$ for some $p \geq 2$. Assume $\frac{1}{2} \leq \sigma<\frac{2}{N-2}$ if $\lambda<0$, or $\frac{1}{2} \leq \sigma<\frac{2}{N}$ if $\lambda>0$. Then the functional $\mathcal{F}(\phi)$ is Gâteaux differentiable and

$$
\mathcal{F}^{\prime}(\phi)=\operatorname{Re} \int_{\mathbb{R}^{N}} \bar{\varphi}(t, x) V(x) u(t, x) d x-2 \frac{d}{d t}\left(\phi^{\prime}(t)\left(\gamma_{2}+\gamma_{1} \omega^{2}(t)\right)\right),
$$

in the sense of distributions, where $\omega(t)=\int_{\mathbb{R}^{N}} V(x)|u(t, x)|^{2} d x$ and $\varphi \in C\left([0, T], L^{2}\right)$ is the solution of the adjoint equation (4.2).

Remarks. (1) Under the assumptions on $u_{0}$ and $V$, it follows from Lemma 2.5 that the solution $u \in L^{\infty}\left((0, T), H^{2}\right)$ of (1.1). Hence, we deduce from the inequality $\|V u\|_{L^{2}} \leq\|V\|_{L^{p}}\|u\|_{L^{\frac{2 p}{p-2}}}$ and $\varphi \in C\left([0, T], L^{2}\right)$ that the right hand side of (1.9) is well-defined.

(2) Because control potential $V$ is unbounded, we cannot follow the method in [11] to obtain sufficiently high regularity of $u$, the solution of the NLS equation (1.1). We resume the idea 
due to T.Kato, (see, [12]), based on the general idea for Schrödinger equations, that two space derivative cost the same as one time derivative.

(3) In contrast with the assumption $\sigma \in \mathbb{N}$ in [11], our results follow for $\frac{1}{2} \leq \sigma<\frac{2}{N-2}$ if $\lambda<0$, or $\frac{1}{2} \leq \sigma<\frac{2}{N}$ if $\lambda>0$.

As an immediate corollary of Theorem 1.2, we derive the precise characterization for the critical points $\phi_{*}$ of functional $\mathcal{F}$. The proof is the same as that of Corollary 4.8 in [11], so we omit it.

Corollary 1.3. Let $u_{*}$ be the solution of (1.1) with control $\phi_{*}$, and $\varphi_{*}$ be the solution of corresponding adjoint equation (4.2). Then $\phi_{*} \in C^{2}(0, T)$ is a classical solution of the following ordinary differential equation

$$
\frac{d}{d t}\left(\phi_{*}^{\prime}(t)\left(\gamma_{2}+\gamma_{1} \omega_{*}^{2}(t)\right)\right)=\frac{1}{2} R e \int_{\mathbb{R}^{N}} \bar{\varphi}_{*}(t, x) V(x) u_{*}(t, x) d x .
$$

subject to the initial data $\phi_{*}(0)=\phi_{0}$ and $\phi_{*}^{\prime}(T)=0$.

This paper is organized as follows: in Section 2, we will collect some preliminaries such as compactness results, global existence and regularity of (1.1). In section 3, we will show Theorem 1.1. In section 4 , we firstly analyze well-posedness of the adjoint equation. Next, the Lipschitz continuity of solution $u=u(\phi)$ with respect to control parameter $\phi$ is obtained. Finally, we give the proof of Theorem 1.2. Some of the steps of the proof follow [11, to avoid repetitions we will mainly focus on the differences with respect to [11].

Notation. Throughout this paper, we use the following notation. $C>0$ will stand for a constant that may different from line to line when it does not cause any confusion. Since we exclusively deal with $\mathbb{R}^{N}$, we often use the abbreviations $L^{r}=L^{r}\left(\mathbb{R}^{N}\right), H^{s}=H^{s}\left(\mathbb{R}^{N}\right)$. Given any interval $I \subset \mathbb{R}$, the norms of mixed spaces $L^{q}\left(I, L^{r}\left(\mathbb{R}^{N}\right)\right)$ and $L^{q}\left(I, H^{s}\left(\mathbb{R}^{N}\right)\right)$ are denoted by $\|\cdot\|_{L^{q}\left(I, L^{r}\right)}$ and $\|\cdot\|_{L^{q}\left(I, H^{s}\right)}$ respectively. We denote by $U(t):=e^{i t \triangle}$ the free Schrödinger propagator, which is isometric on $H^{s}$ for every $s \geq 0$, see [12]. We recall that a pair of exponents $(q, r)$ is Schrödinger-admissible if $\frac{2}{q}=N\left(\frac{1}{2}-\frac{1}{r}\right)$ and $2 \leq r \leq \frac{2 N}{N-2},(2 \leq r \leq \infty$ if $N=1$; $2 \leq r<\infty$ if $N=2$ ).

\section{Preliminaries}

In this section, we recall some useful results. First, we recall the following two compactness lemmas which is vital in our paper, see 12 for detailed presentation.

Lemma 2.1. [12] Let $X \hookrightarrow Y$ be two Banach spaces, $I$ be a bounded, open interval of $\mathbb{R}$, and $\left(u_{n}\right)_{n \in \mathbb{N}}$ be a bounded sequence in $C(\bar{I}, Y)$. Assume that $u_{n}(t) \in X$ for all $(n, t) \in \mathbb{N} \times I$ and that $\sup \left\{\left\|u_{n}(t)\right\|_{X},(n, t) \in \mathbb{N} \times I\right\}=K<\infty$. Assume further that $u_{n}$ is uniformly equicontinuous in 
$Y$. If $X$ is reflexive, then there exist a function $u \in C(\bar{I}, Y)$ which is weakly continuous $\bar{I} \rightarrow X$ and some subsequence $\left(u_{n_{k}}\right)_{k \in \mathbb{N}}$ such that for every $t \in \bar{I}, u_{n_{k}}(t) \rightarrow u(t)$ in $X$ as $k \rightarrow \infty$.

Lemma 2.2. [12] Let $I$ be a bounded interval of $\mathbb{R}$, and $\left(u_{n}\right)_{n \in \mathbb{N}}$ be a bounded sequence of $L^{\infty}\left(I, H_{0}^{1}\right) \cap W^{1, \infty}\left(I, H^{-1}\right)$. Then, there exist $u \in L^{\infty}\left(I, H_{0}^{1}\right) \cap W^{1, \infty}\left(I, H^{-1}\right)$ and some subsequence $\left(u_{n_{k}}\right)_{k \in \mathbb{N}}$ such that for every $t \in \bar{I}, u_{n_{k}}(t) \rightarrow u(t)$ in $H_{0}^{1}$ as $k \rightarrow \infty$.

In the following lemma, we establish some existence results of equation (1.1).

Lemma 2.3. Let $u_{0} \in H^{1}$ and $V \in L^{p}+L^{\infty}$ for some $p \geq 1, p>N / 2$. Assume $0<\sigma<\frac{2}{N-2}$ if $\lambda<0$, or $0<\sigma<\frac{2}{N}$ if $\lambda>0$. For any given $T>0, \phi \in H^{1}(0, T)$, there exists a unique mild solution $u \in C\left([0, T], H^{1}\right)$ of problem (1.1).

Proof. When $\phi$ is a constant, the author in [12] showed that the solution of (1.1) is local wellposedness. For our case, since $\phi \in H^{1}(0, T) \hookrightarrow L^{\infty}(0, T)$, we only need to take the $L^{\infty}$ norm of $\phi$ when the term $\phi V u$ has to be estimated in some norms. Keeping this in mind and applying the method in [12], one can show the local well-posedness of (1.1). Hence, in order to prove this lemma, it suffices to show

$$
\|u(t)\|_{H^{1}} \leq C\left(T,\left\|u_{0}\right\|_{H^{1}}, \phi\right) .
$$

Indeed, we deduce from (1.4) and mass conservation that

$$
\left\|E^{\prime}\right\|_{L^{2}(0, T)} \leq C\left\|\phi^{\prime}\right\|_{L^{2}(0, T)}\left(\left\|V_{1}\right\|_{L^{p}}\|u\|_{L^{\frac{2 p}{p-1}}}^{2}+\left\|V_{2}\right\|_{L^{\infty}}\left\|u_{0}\right\|_{L^{2}}^{2}\right) .
$$

This implies

$$
\begin{aligned}
E(t) & =E(0)+\int_{0}^{t} E^{\prime}(s) d s \leq E(0)+\left(T \int_{0}^{T}\left(E^{\prime}(s)\right)^{2} d s\right)^{1 / 2} \\
& \leq E(0)+C T^{1 / 2}\left\|\phi^{\prime}\right\|_{L^{2}(0, T)}\left(\left\|V_{1}\right\|_{L^{p}}\|u\|_{L^{\frac{2 p}{p-1}}}^{2}+\left\|V_{2}\right\|_{L^{\infty}}\left\|u_{0}\right\|_{L^{2}}^{2}\right) .
\end{aligned}
$$

When $\lambda \leq 0$, it follows from (1.3) that

$$
\begin{aligned}
\|\nabla u(t)\|_{L^{2}}^{2} \leq & C E(t)+C\|\phi\|_{L^{\infty}(0, T)} \int V|u|^{2} d x \\
\leq & C E(0)+C T^{1 / 2}\left\|\phi^{\prime}\right\|_{L^{2}(0, T)}\left(\left\|V_{1}\right\|_{L^{p}}\|u\|_{L^{\frac{2 p}{p-1}}}^{2}+\left\|V_{2}\right\|_{L^{\infty}}\left\|u_{0}\right\|_{L^{2}}^{2}\right) \\
& +C\|\phi\|_{L^{\infty}(0, T)}\left(\left\|V_{1}\right\|_{L^{p}}\|u\|_{L^{\frac{2 p}{p-1}}}^{2}+\left\|V_{2}\right\|_{L^{\infty}}\left\|u_{0}\right\|_{L^{2}}^{2}\right),
\end{aligned}
$$

which, together with the embedding $H^{1} \hookrightarrow L^{\frac{2 p}{p-1}}$ and Young's inequality with $\varepsilon$, implies (2.1).

When $\lambda>0$, by the same argument as above, we have

$$
\begin{aligned}
\|\nabla u(t)\|_{L^{2}}^{2} \leq & C E(0)+C T^{1 / 2}\left\|\phi^{\prime}\right\|_{L^{2}(0, T)}\left(\left\|V_{1}\right\|_{L^{p}}\|u\|_{L^{\frac{2 p}{p-1}}}^{2}+\left\|V_{2}\right\|_{L^{\infty}}\left\|u_{0}\right\|_{L^{2}}^{2}\right) \\
& +C\|\phi\|_{L^{\infty}(0, T)}\left(\left\|V_{1}\right\|_{L^{p}}\|u\|_{L^{\frac{2 p}{p-1}}}^{2}+\left\|V_{2}\right\|_{L^{\infty}}\left\|u_{0}\right\|_{L^{2}}^{2}\right)+C\|u\|_{L^{2 \sigma+2}}^{2 \sigma+2},
\end{aligned}
$$


It follows from Gagliardo-Nirenberg's inequality that

$$
\|u\|_{L^{2 \sigma+2}}^{2 \sigma+2} \leq C\|u\|_{H^{1}}^{N \sigma}\|u\|_{L^{2}}^{2 \sigma+2-N \sigma} .
$$

Since $N \sigma<2$, (2.1) follows from Young's inequality with $\varepsilon$.

Lemma 2.4. [12] Let $J \ni 0$ be a bounded interval, $(\gamma, \rho)$ be an admissible pair and consider $f \in L^{\infty}\left(J, L^{2}\right)$ such that $f_{t} \in L^{\gamma^{\prime}}\left(J, L^{\rho^{\prime}}\right)$. If

$$
v(t)=i \int_{0}^{t} U(t-s) f(s) d s \quad \text { for all } t \in J
$$

then $v \in L^{\infty}\left(J, H^{2}\right) \cap C^{1}\left(J, L^{2}\right) \cap W^{1, a}\left(J, L^{b}\right)$ for every admissible pair $(a, b)$ and

$$
\|\Delta v\|_{L^{\infty}\left(J, L^{2}\right)} \leq\|f\|_{L^{\infty}\left(J, L^{2}\right)}+\|f(0)\|_{L^{2}}+C\|f\|_{L^{\gamma^{\prime}\left(J, L^{\rho^{\prime}}\right)}}
$$

where $C$ is independent of $J$ and $f$.

Lemma 2.5. Let $u_{0} \in H^{2}, \phi \in H^{1}(0, T)$ and $V, \nabla V \in L^{p}+L^{\infty}$ for some $p \geq 2, p>N / 2$. Assume $0<\sigma<\frac{2}{N-2}$ if $\lambda<0$ or $0<\sigma<\frac{2}{N}$ if $\lambda>0$. Then the mild solution of (1.1) satisfies $u \in L^{\infty}\left((0, T), H^{2}\right)$.

This lemma can be proved by applying Remarks 5.3.3 and 5.3.5 in [12]. When $0<\sigma<\frac{2}{N}$, for this lemma, it suffices to require $V \in L^{p}+L^{\infty}$ for some $p \geq 1, p>N / 2$, see Remark 5.3.5 in 12$]$.

\section{Existence of Minimizers}

Our goal in this section is to prove Theorem 1.1.

Proof of Theorem 1.1. The proof proceeds in three steps.

Step 1. Estimates of $\left(u_{n}, \phi_{n}\right)_{n \in \mathbb{N}}$. Let $\phi \in H^{1}(0, T)$, there exists a unique mild solution $u \in C\left([0, T], H^{1}\right)$ of (1.1) by Lemma 2.3. Hence, the set $\Lambda(0, T)$ is nonempty, and there exists a minimizing sequence $\left(u_{n}, \phi_{n}\right)_{n \in \mathbb{N}}$ such that

$$
\lim _{n \rightarrow \infty} F\left(u_{n}, \phi_{n}\right)=F_{*}
$$

We deduce from $\gamma_{2}>0$ that there exists a constant $C$ such that for every $n \in \mathbb{N}$

$$
\int_{0}^{T}\left(\phi_{n}^{\prime}(t)\right)^{2} d t \leq C<+\infty
$$

By using the embedding $H^{1}(0, T) \hookrightarrow C[0, T]$ and $\phi_{n}(0) \in B_{2}$, we have

$$
\phi_{n}(t)=\phi_{n}(0)+\int_{0}^{t} \phi_{n}^{\prime}(s) d s \leq M_{2}+\left(T \int_{0}^{T}\left(\phi_{n}^{\prime}(s)\right)^{2} d s\right)^{1 / 2}<+\infty .
$$


This implies the sequence $\left(\phi_{n}\right)_{n \in \mathbb{N}}$ is bounded in $L^{\infty}(0, T)$, so is in $H^{1}(0, T)$. Thus, there exist a subsequence, which we still denote by $\left(\phi_{n}\right)_{n \in \mathbb{N}}$, and $\phi_{*} \in H^{1}(0, T)$ such that

$$
\phi_{n} \rightarrow \phi_{*} \text { in } H^{1}(0, T) \text { and } \phi_{n} \rightarrow \phi_{*} \text { in } L^{2}(0, T) \text { as } n \rightarrow \infty \text {. }
$$

On the other hand, we deduce from (1.4) and mass conservation that

$$
\left\|E_{n}^{\prime}\right\|_{L^{2}(0, T)} \leq C\left\|\phi_{n}^{\prime}\right\|_{L^{2}(0, T)}\|V\|_{L^{\infty}}\left\|u_{0}\right\|_{L^{2}}^{2} .
$$

Using the same argument as Lemma 2.3 and $u_{n}(0) \in B_{2}$, we derive

$$
\left\|u_{n}\right\|_{L^{\infty}\left((0, T), H^{1}\right)} \leq C .
$$

Combining this estimate and the fact that $u_{n}$ is the solution of (1.1), we have

$$
\left\|\left(u_{n}\right)_{t}\right\|_{L^{\infty}\left((0, T), H^{-1}\right)} \leq C .
$$

Step 2. Passage to the limit. By applying (3.2), (3.3), and Lemma 2.2, we deduce that there exist $u_{*} \in L^{\infty}\left((0, T), H^{1}\right) \cap W^{1, \infty}\left((0, T), H^{-1}\right)$ and a subsequence, still denoted by $\left(u_{n}\right)_{n \in \mathbb{N}}$, such that, for all $t \in[0, T]$,

$$
u_{n}(t) \rightarrow u_{*}(t) \text { in } H^{1} \text { as } n \rightarrow \infty .
$$

From the embedding $W^{1, \infty}\left((0, T), H^{-1}\right) \hookrightarrow C^{0,1}\left([0, T], H^{-1}\right)$ (see [12], Remark 1.3.11) and the inequality $\|u\|_{L^{2}}^{2} \leq\|u\|_{H^{1}}\|u\|_{H^{-1}}$, we derive for every $u \in L^{\infty}\left((0, T), H^{1}\right) \cap W^{1, \infty}\left((0, T), H^{-1}\right)$

$$
\|u(t)-u(s)\|_{L^{2}} \leq C|t-s|^{\frac{1}{2}}, \text { for all } t, s \in(0, T) .
$$

Next, we note that for all $z_{1}, z_{2} \in \mathbb{C}$, it holds

$$
\left.|| z_{1}\right|^{2 \sigma} z_{1}-\left|z_{2}\right|^{2 \sigma} z_{2}\left|\leq C\left(\left|z_{1}\right|^{2 \sigma}+\left|z_{2}\right|^{2 \sigma}\right)\right| z_{1}-z_{2} \mid .
$$

It follows from (2.4), (3.2), (3.5), (3.6), Hölder's inequality that

$$
\begin{aligned}
\left\|\left|u_{n}(t)\right|^{2 \sigma} u_{n}(t)-\left|u_{n}(s)\right|^{2 \sigma} u_{n}(s)\right\|_{L^{r^{\prime}}} & \leq C\left(\left\|u_{n}(t)\right\|_{L^{r}}^{2 \sigma}+\left\|u_{n}(s)\right\|_{L^{r}}^{2 \sigma}\right)\left\|u_{n}(t)-u_{n}(s)\right\|_{L^{r}} \\
& \leq C\left\|u_{n}(t)-u_{n}(s)\right\|_{L^{2}}^{a} \leq C|t-s|^{\frac{a}{2}}
\end{aligned}
$$

where $r=2 \sigma+2$ and $a=1-N\left(\frac{1}{2}-\frac{1}{2 \sigma+2}\right)$. This implies $\left(\left|u_{n}\right|^{2 \sigma} u_{n}\right)_{n \in \mathbb{N}}$ is a bounded sequence in $C^{0, \frac{a}{2}}\left([0, T], L^{r^{\prime}}\right)$. Therefore, we deduce from Lemma 2.1 that there exist a subsequence, still denoted by $\left(\left|u_{n}\right|^{2 \sigma} u_{n}\right)_{n \in \mathbb{N}}$, and $f \in C^{0, \frac{a}{2}}\left([0, T], L^{r^{\prime}}\right)$ such that, for all $t \in[0, T]$,

$$
\left|u_{n}(t)\right|^{2 \sigma} u_{n}(t) \rightarrow f(t) \text { in } L^{r^{\prime}} \text { as } n \rightarrow \infty .
$$

On the other hand, it follows from $\left(u_{n}, \phi_{n}\right) \in \Lambda(0, T)$ that for every $\omega \in C_{c}^{\infty}\left(\mathbb{R}^{N}\right)$ and for every $\eta \in \mathcal{D}(0, T)$,

$$
\int_{0}^{T}\left[-\left\langle i u_{n}, \omega\right\rangle_{H^{-1}, H_{0}^{1}} \eta^{\prime}(t)+\left\langle\Delta u_{n}+\left|u_{n}\right|^{2 \sigma} u_{n}+\phi_{n}(t) V u_{n}, \omega\right\rangle_{H^{-1}, H_{0}^{1}} \eta(t)\right] d t=0 .
$$


Applying (3.1), (3.4), (3.8), and the dominated convergence theorem, we deduce easily that

$$
\int_{0}^{T}\left[-\left\langle i u_{*}, \omega\right\rangle_{H^{-1}, H_{0}^{1}} \eta^{\prime}(t)+\left\langle\Delta u_{*}+f+\phi_{*}(t) V u_{*}, \omega\right\rangle_{H^{-1}, H_{0}^{1}} \eta(t)\right] d t=0 .
$$

This implies that $u_{*}$ satisfies

$$
i \frac{d}{d t} u_{*}+\Delta u_{*}+f+\phi_{*}(t) V u_{*}=0 \text { for a.e. } t \in[0, T] .
$$

We next show $\left|u_{*}(t, x)\right|^{2 \sigma} u_{*}(t, x)=f(t, x)$ for a.e. $(t, x) \in[0, T] \times \mathbb{R}^{N}$. It suffices to show that for any given $t \in[0, T]$

$$
\int_{\mathbb{R}^{N}}\left|u_{*}(t, x)\right|^{2 \sigma}(x) u_{*}(t, x) \varphi(x) d x=\int_{\mathbb{R}^{N}} f(t, x) \varphi(x) d x \quad \text { for any } \varphi \in C_{c}^{\infty}\left(\mathbb{R}^{N}\right) .
$$

Let us prove (3.10) by contradiction. If not, there exists $\varphi_{0} \in C_{c}^{\infty}\left(\mathbb{R}^{N}\right)$ such that

$$
\int_{\mathbb{R}^{N}}\left|u_{*}(t, x)\right|^{2 \sigma}(x) u_{*}(t, x) \varphi_{0}(x) d x \neq \int_{\mathbb{R}^{N}} f(t, x) \varphi_{0}(x) d x .
$$

It follows from (3.8) that

$$
\int_{\mathbb{R}^{N}}\left|u_{n}(t, x)\right|^{2 \sigma}(x) u_{n}(t, x) \varphi_{0}(x) d x \rightarrow \int_{\mathbb{R}^{N}} f(t, x) \varphi_{0}(x) d x \text { as } n \rightarrow \infty .
$$

On the other hand, we deduce from (3.4) that there exists a subsequence, still denoted by $\left(u_{n}(t)\right)_{n \in \mathbb{N}}$ such that $u_{n}(t) \rightarrow u_{*}(t)$ in $L_{l o c}^{2 \sigma+2}\left(\mathbb{R}^{N}\right)$ and $\left|u_{n}(t)\right|^{2 \sigma} \rightarrow\left|u_{*}(t)\right|^{2 \sigma}$ in $L_{l o c}^{\frac{2 \sigma+2}{2 \sigma}}\left(\mathbb{R}^{N}\right)$. Combining this, (3.2) and (3.4), we derive

$$
\begin{aligned}
& \left.\left|\int_{\mathbb{R}^{N}}\right| u_{n}(t, x)\right|^{2 \sigma} u_{n}(t, x) \varphi_{0}(x) d x-\int_{\mathbb{R}^{N}}\left|u_{*}(t, x)\right|^{2 \sigma} u_{*}(t, x) \varphi_{0}(x) d x \mid \\
\leq & \left\|u_{n}(t)\right\|_{L^{2 \sigma+2}}^{2 \sigma}\left\|u_{n}(t)-u_{*}(t)\right\|_{L^{2 \sigma+2}(\Omega)}\left\|\varphi_{0}\right\|_{L^{2 \sigma+2}}+\left\|u_{*}(t)\right\|_{L^{2 \sigma+2}}\left\|u_{n}(t)-u_{*}(t)\right\|_{L^{\frac{2 \sigma+2}{2 \sigma}}(\Omega)}\left\|\varphi_{0}\right\|_{L^{2 \sigma+2}} \\
& \stackrel{n \rightarrow \infty}{\longrightarrow} 0,
\end{aligned}
$$

where $\Omega$ is the compact support of $\varphi_{0}$. This is a contradiction with (3.11) and (3.12).

In summary, $u_{*} \in L^{\infty}\left((0, T), H^{1}\right) \cap W^{1, \infty}\left((0, T), H^{-1}\right)$ and satisfies

$$
i \frac{d}{d t} u_{*}+\Delta u_{*}+\lambda\left|u_{*}\right|^{2 \sigma} u_{*}+\phi_{*}(t) V u_{*}=0, \text { for a.e. } t \in[0, T] .
$$

By using the classical argument based on Strichartz's estimate, we can obtain the uniqueness of the weak solution $u_{*}$ of (1.1). Arguing as the proof of Theorem 3.3.9 in [12], it follows that $u_{*}$ is indeed a mild solution of (1.1) and $u_{*} \in C\left((0, T), H^{1}\right) \cap C^{1}\left((0, T), H^{-1}\right)$.

Step 3. Conclusion. In order to conclude that the pair $\left(u_{*}, \phi_{*}\right) \in \Lambda(0, T)$ is indeed a minimizer of optimal control problem (1.8), we need only show

$$
F_{*}=\lim _{n \rightarrow \infty} F\left(u_{n}, \phi_{n}\right) \geq F\left(u_{*}, \phi_{*}\right)
$$


Indeed, in view of the assumption on operator $A$, there exists $R>0$, such that for every $n \in \mathbb{N}, \operatorname{supp}_{x \in \mathbb{R}^{3}}(A u(T, x)) \subseteq B(R)$. Therefore, we deduce from $u_{n}(T) \rightarrow u_{*}(T)$ in $L_{l o c}^{2}$ and $A u_{n}(T) \rightarrow A u_{*}(T)$ in $L^{2}$ that

$$
\begin{aligned}
& \left|\left\langle u_{n}(T), A u_{n}(T)\right\rangle-\left\langle u_{*}(T), A u_{*}(T)\right\rangle\right| \\
\leq & \left|\left\langle u_{n}(T)-u_{*}(T), A u_{n}(T)\right\rangle\right|+\left|\left\langle u_{*}(T), A\left(u_{n}(T)-u_{*}(T)\right)\right\rangle\right| \rightarrow 0 \text { as } n \rightarrow \infty .
\end{aligned}
$$

The same argument as Lemma 2.5 in [11, we have

$$
\liminf _{n \rightarrow \infty} \int_{0}^{T}\left(\phi_{n}^{\prime}(t)\right)^{2} \omega_{n}^{2}(t) d t \geq \int_{0}^{T}\left(\phi_{*}^{\prime}(t)\right)^{2} \omega_{*}^{2}(t) d t
$$

where

$$
\omega_{n}(t)=\int_{\mathbb{R}^{3}} V(x)\left|u_{n}(t, x)\right|^{2} d x, \quad \omega_{*}(t)=\int_{\mathbb{R}^{3}} V(x)\left|u_{*}(t, x)\right|^{2} d x .
$$

It follows from the weak lower semicontinuity of the norm that

$$
\liminf _{n \rightarrow \infty} \int_{0}^{T}\left(\phi_{n}^{\prime}(t)\right)^{2} d t \geq \int_{0}^{T}\left(\phi_{*}^{\prime}(t)\right)^{2} d t .
$$

Collecting (3.15)-(3.17), we derive (3.14). This completes the proof.

\section{Rigorous characterization of a minimizer}

In order to obtain a rigorous characterization of a minimizer $\left(u_{*}, \phi_{*}\right) \in \Lambda(0, T)$, we need to derive the first order optimality conditions for our optimal control problem (1.8). For this aim, we firstly formally calculate the derivative of the objective functional $F(u, \phi)$ and analyze the resulting adjoint problem in the next subsection.

\subsection{Derivation and analysis of the adjoint equation.}

To begin with, we rewrite equation (1.1) in a more abstract form, i.e.,

$$
P(u, \phi)=i u_{t}+\Delta u+\lambda|u|^{2 \sigma} u+\phi(t) V(x) u=0 .
$$

Thus, formally computation yields

$$
\partial_{u} P(u, \phi) \varphi=i \varphi_{t}+\Delta \varphi+\phi(t) V(x) \varphi+\lambda(\sigma+1)|u|^{2 \sigma} \varphi+\lambda|u|^{2 \sigma-2} u^{2} \bar{\varphi},
$$

where $\varphi \in L^{2}$. Similarly, we have

$$
\partial_{\phi} P(u, \phi)=V(x) u .
$$

The analogue argument as Section 3.1 in [11, we can derive the following adjoint equation:

$$
\left\{\begin{array}{l}
i \varphi_{t}+\Delta \varphi+\phi(t) V(x) \varphi+\lambda(\sigma+1)|u|^{2 \sigma} \varphi+\lambda|u|^{2 \sigma-2} u^{2} \bar{\varphi}=\frac{\delta F(u, \phi)}{\delta u(t)} \\
\varphi(T)=i \frac{\delta F(u, \phi)}{\delta u(T)}
\end{array}\right.
$$


where $\frac{\delta F(u, \phi)}{\delta u(t)}$ and $\frac{\delta F(u, \phi)}{\delta u(T)}$ denote the first variation of $F(u, \phi)$ with respect to $u(t)$ and $u(T)$ respectively. By straightforward computations, we have

$$
\begin{aligned}
\frac{\delta F(u, \phi)}{\delta u(t)} & =4 \gamma_{1}\left(\phi^{\prime}(t)\right)^{2}\left(\int_{\mathbb{R}^{N}} V(x)|u(t, x)|^{2} d x\right) V(x) u(t, x) \\
& =4 \gamma_{1}\left(\phi^{\prime}(t)\right)^{2} \omega(t) V(x) u(t, x),
\end{aligned}
$$

and

$$
\frac{\delta F(u, \phi)}{\delta u(T)}=4\langle u(T), A u(T)\rangle_{L^{2}} A u(T) .
$$

Thus, equation (4.2) defines a Cauchy problem for $\varphi$ with data $\varphi(T) \in L^{2}$, one can solve (4.2) backwards in time.

In the following proposition, we will analyze the existence of solutions to (4.2).

Proposition 4.1. Let $N \leq 3, u_{0} \in H^{2}$ and $V, \nabla V \in L^{p}+L^{\infty}$ for some $p \geq 2$. Assume $0<\sigma<\frac{2}{N-2}$ if $\lambda<0$ or $0<\sigma<\frac{2}{N}$ if $\lambda>0$. Then, for every $T>0$, equation (4.2) admits a unique mild solution $\varphi \in C\left([0, T], L^{2}\right)$.

Proof. Under our assumptions on $V, u_{0}$, and $A$, we deduce from $H^{2} \hookrightarrow L^{\infty}$ and Lemma 2.5 that $|u|^{2 \sigma},|u|^{2 \sigma-2} u^{2} \in L^{\infty}, \frac{\delta F(u, \phi)}{\delta u(t)} \in L^{1}\left((0, T), L^{\frac{2 p}{p+1}}\right), \frac{\delta F(u, \phi)}{\delta u(T)} \in L^{2}$. Since $V$ is an unbounded potential, it cannot be treated as a perturbation. Applying consequently Theorem 4.6.4 and Corollary 4.6.5 in [12], we can obtain the local well-posedness. The global existence can be derived by the classical argument for Schrödinger equations and Gronwall's inequality.

\subsection{Lipschitz continuity with respect to the control.}

This subsection is devoted to derive the solution of (1.1) depends Lipschitz continuously on the control parameter $\phi$, which is vital for investigating the differentiability of unconstrained functional $\mathcal{F}$. To begin with, we study the continuous dependence of the solutions $u=u(\phi)$ with respect to the control parameter $\phi$. Our result is as follows.

Proposition 4.2. Let $N \leq 3, V, \nabla V \in L^{p}+L^{\infty}$ and $V \in L^{2 p}$ for some $p \geq 2$. Assume $0<\sigma<\frac{2}{N-2}$ if $\lambda<0$ or $0<\sigma<\frac{2}{N}$ if $\lambda>0$. Let $u, \tilde{u} \in L^{\infty}\left((0, T), H^{2}\right)$ be two mild solutions of (1.1) with the same initial data $u_{0} \in H^{2}$, corresponding to control parameters $\phi, \tilde{\phi} \in H^{1}(0, T)$ respectively. Given a constant $M>0$, if

$$
\|\phi\|_{H^{1}(0, T)},\|\tilde{\phi}\|_{H^{1}(0, T)},\|u(t)\|_{H^{2}},\|\tilde{u}(t)\|_{H^{2}} \leq M,
$$

then, there exist $\tau=\tau(M)>0$ and a constant $C=C(M)$ such that

$$
\|u-\tilde{u}\|_{L^{\infty}\left(I_{t}, H^{2}\right)} \leq C\left(\|u(t)-\tilde{u}(t)\|_{H^{2}}+\|\phi-\tilde{\phi}\|_{L^{2}\left(I_{t}\right)}\right),
$$

where $I_{t}:=[t, t+\tau] \cap[0, T]$. In particular, the solution $u(\phi)$ depends continuously on control parameter $\phi \in H^{1}(0, T)$. 
Proof. To simplify notation, let us assume $t+\tau \leq T$. Applying Lemmas 2.3 and 2.4, there is a $\tau>0$ depending only on $M$, such that $\left.u\right|_{I_{t}}$ is a fixed point of the operator

$$
\Phi(u):=U(\cdot-t) u(t)+i \int_{t}^{\cdot} U(\cdot-s)\left(\lambda|u(s)|^{2 \sigma} u(s)+\phi(s) V u(s)\right) d s,
$$

which maps the Banach space

$$
Y=\left\{u \in L^{\infty}\left(I_{t}, H^{2}\right), \quad\|u\|_{L^{\infty}\left(I_{t}, H^{2}\right)} \leq 2 M\right\}
$$

into itself. The same holds for $\tilde{u}$, we consequently derive

$$
\begin{aligned}
& \tilde{u}(s)-u(s)=U(s-t)(\tilde{u}(t)-u(t)) \\
& \quad+i \int_{t}^{s} U(s-r)\left(\lambda\left(|\tilde{u}|^{2 \sigma} \tilde{u}-|u|^{2 \sigma} u\right)+V(\tilde{u} \tilde{\phi}-u \phi)\right)(r) d r
\end{aligned}
$$

where $s \in[t, t+\tau]$. In the following, we set $r=2 \sigma+2$ and $\rho=\frac{2 p}{p-1}$, taking $q$ and $\gamma$ such that $(q, r)$ and $(\gamma, \rho)$ are two admissible pairs. Applying Strichartz's estimate to (4.6), the embedding theorems $\tilde{\phi}, \phi \in H^{1}(0, T) \hookrightarrow L^{\infty}(0, T)$ and $\tilde{u}(t, \cdot), u(t, \cdot) \in H^{2}\left(\mathbb{R}^{N}\right) \hookrightarrow L^{\infty}\left(\mathbb{R}^{N}\right)$ when $N \leq 3$, Hölder's inequality, (3.6), we derive

$$
\begin{aligned}
& \|\tilde{u}-u\|_{L^{\infty}\left(I_{t}, L^{2}\right)} \\
& \left.\leq C\|\tilde{u}(t)-u(t)\|_{L^{2}}+C\left\||\tilde{u}|^{2 \sigma} \tilde{u}-|u|^{2 \sigma} u\right\|_{L^{q^{\prime}\left(I_{t}, L^{r^{\prime}}\right)}}+C \| V(\tilde{u} \tilde{\phi}-u \phi)\right) \|_{L^{\gamma^{\prime}}\left(I_{t}, L^{\rho^{\prime}}\right)} \\
& \leq C\|\tilde{u}(t)-u(t)\|_{L^{2}}+C \tau^{\frac{q-q^{\prime}}{q q^{\prime}}}\left(\|\tilde{u}\|_{L^{\infty}\left(I_{t}, L^{r}\right)}^{2 \sigma}+C\|u\|_{L^{\infty}\left(I_{t}, L^{r}\right)}^{2 \sigma}\right)\|\tilde{u}-u\|_{L^{q}\left(I_{t}, L^{r}\right)} \\
& +C\|V\|_{L^{p}}\|\tilde{\phi}\|_{L^{\infty}}\|\tilde{u}-u\|_{L^{\gamma^{\prime}\left(I_{t}, L^{\rho}\right)}}+\|V\|_{L^{p}}\|\tilde{\phi}-\phi\|_{L^{\gamma^{\prime}}\left(I_{t}\right)}\|u\|_{L^{\infty}\left(I_{t}, L^{\rho}\right)} \\
& \leq C\|\tilde{u}(t)-u(t)\|_{L^{2}}+C \tau^{\frac{1}{q^{\prime}}}\|\tilde{u}-u\|_{L^{\infty}\left(I_{t}, H^{2}\right)}+C \tau^{\frac{1}{\gamma^{\prime}}}\|\tilde{u}-u\|_{L^{\infty}\left(I_{t}, H^{2}\right)}+C\|\tilde{\phi}-\phi\|_{H^{1}\left(I_{t}\right)} \text {. }
\end{aligned}
$$

Set $f_{1}(t)=\lambda\left(|\tilde{u}|^{2 \sigma} \tilde{u}-|u|^{2 \sigma} u\right)(t)$ and $f_{2}(t)=V(\tilde{u} \tilde{\phi}-u \phi)(t)$, we deduce from Lemma 2.4 that

$$
\begin{aligned}
\|\Delta(\tilde{u}-u)\|_{L^{\infty}\left(I_{t}, L^{2}\right)} \leq & \|\Delta(\tilde{u}(t)-u(t))\|_{L^{2}}+C\left\|f_{1}(t)+f_{2}(t)\right\|_{L^{2}}+\left\|f_{1}+f_{2}\right\|_{L^{\infty}\left(I_{t}, L^{2}\right)} \\
& +C\left\|\left(f_{1}\right)_{t}\right\|_{L^{q^{\prime}\left(I_{t}, L^{r^{\prime}}\right)}}+C\left\|\left(f_{2}\right)_{t}\right\|_{L^{\gamma^{\prime}}\left(I_{t}, L^{\rho^{\prime}}\right)} .
\end{aligned}
$$

Let us estimate these terms. By the similar argument as (4.7), we obtain

$$
\begin{aligned}
\left\|f_{1}(t)+f_{2}(t)\right\|_{L^{2}} & \leq C\left\|\left(|\tilde{u}|^{2 \sigma}+|u|^{2 \sigma}\right)(t)(\tilde{u}-u)(t)\right\|_{L^{2}}+\|V \tilde{\phi}(t)(\tilde{u}-u)(t)\|_{L^{2}}+\|V u(t)(\tilde{\phi}-\phi)(t)\|_{L^{2}} \\
& \leq C\|\tilde{u}(t)-u(t)\|_{L^{2}}+\tilde{\phi}(t)\|V\|_{L^{p}}\|(\tilde{u}-u)(t)\|_{L^{\frac{2 p}{p-2}}}+|(\tilde{\phi}-\phi)(t)|\|V u(t)\|_{L^{2}} \\
& \leq C\|\tilde{u}(t)-u(t)\|_{L^{2}}+C\|\tilde{u}(t)-u(t)\|_{H^{2}}+C\|\tilde{\phi}-\phi\|_{H^{1}\left(I_{t}\right)} ;
\end{aligned}
$$

When $2<p<\infty, 2<\frac{2 p}{p-2}<\infty$, we deduce from interpolation inequality and Young's inequality with $\varepsilon$ that

$$
\begin{aligned}
& \left\|f_{1}+f_{2}\right\|_{L^{\infty}\left(I_{t}, L^{2}\right)} \\
& \leq C\left\|\left(|\tilde{u}|^{2 \sigma}+|u|^{2 \sigma}\right)(\tilde{u}-u)\right\|_{L^{\infty}\left(I_{t}, L^{2}\right)}+\|V \tilde{\phi}(\tilde{u}-u)\|_{L^{\infty}\left(I_{t}, L^{2}\right)}+\|V u(\tilde{\phi}-\phi)\|_{L^{\infty}\left(I_{t}, L^{2}\right)} \\
& \leq C\|\tilde{u}-u\|_{L^{\infty}\left(I_{t}, L^{2}\right)}+C\|V\|_{L^{p}}\|\tilde{u}-u\|_{L^{\infty}\left(I_{t}, L^{\frac{2 p}{p-2}}\right)}+\|\tilde{\phi}-\phi\|_{H^{1}\left(I_{t}\right)}\|V\|_{L^{p}}\|u\|_{L^{\infty}\left(I_{t}, H^{2}\right)} \\
& \leq C\|\tilde{u}-u\|_{L^{\infty}\left(I_{t}, L^{2}\right)}+\varepsilon\|\tilde{u}-u\|_{L^{\infty}\left(I_{t}, H^{2}\right)}+C\|\tilde{\phi}-\phi\|_{H^{1}\left(I_{t}\right)} .
\end{aligned}
$$


When $p=2$, by the similar argument as above, we have

$$
\begin{aligned}
\|V \tilde{\phi}(\tilde{u}-u)\|_{L^{\infty}\left(I_{t}, L^{2}\right)} & \leq\|V\|_{L^{4}}\|\tilde{\phi}(\tilde{u}-u)\|_{L^{\infty}\left(I_{t}, L^{4}\right)} \\
& \leq C\|\tilde{u}-u\|_{L^{\infty}\left(I_{t}, L^{2}\right)}+\varepsilon\|\tilde{u}-u\|_{L^{\infty}\left(I_{t}, H^{2}\right)}
\end{aligned}
$$

When $p=\infty$,

$$
\|V \tilde{\phi}(\tilde{u}-u)\|_{L^{\infty}\left(I_{t}, L^{2}\right)} \leq\|V\|_{L^{\infty}}\|\tilde{\phi}(\tilde{u}-u)\|_{L^{\infty}\left(I_{t}, L^{2}\right)} .
$$

After some fundamental computations, by the similar argument as (4.7), we obtain

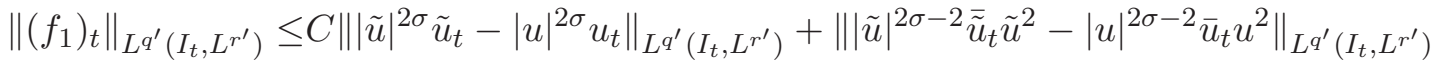

$$
\begin{aligned}
& \leq C\left\|\tilde{u}_{t}-u_{t}\right\|_{L^{q^{\prime}\left(I_{t}, L^{2}\right)}}+\left\||\tilde{u}|^{2 \sigma-2} \tilde{u}^{2}\left(\overline{\tilde{u}}_{t}-\bar{u}_{t}\right)\right\|_{L^{q^{\prime}}\left(I_{t}, L^{r^{\prime}}\right)} \\
& +\left\|\left(|\tilde{u}|^{2 \sigma-2} \tilde{u}^{2}-|u|^{2 \sigma-2} u^{2}\right) \bar{u}_{t}\right\|_{L^{q^{\prime}}\left(I_{t}, L^{r^{\prime}}\right)} \\
& \leq C\left\|\tilde{u}_{t}-u_{t}\right\|_{L^{q^{\prime}}\left(I_{t}, L^{2}\right)}+C\left\|(\tilde{u}-u)\left(|\tilde{u}|^{2 \sigma-1}+|u|^{2 \sigma-1}\right) \bar{u}_{t}\right\|_{L^{q^{\prime}\left(I_{t}, L^{r^{\prime}}\right)}} \\
& \leq C\left\|\tilde{u}_{t}-u_{t}\right\|_{L^{q^{\prime}\left(I_{t}, L^{2}\right)}}+\tau^{\frac{1}{q^{\prime}}}\|\tilde{u}-u\|_{L^{\infty}\left(I_{t}, H^{2}\right)},
\end{aligned}
$$

where

$$
\begin{aligned}
& \left\|\tilde{u}_{t}-u_{t}\right\|_{L^{q^{\prime}}\left(I_{t}, L^{2}\right)} \\
\leq & C\|\Delta(\tilde{u}-u)\|_{L^{q^{\prime}\left(I_{t}, L^{2}\right)}}+C\|V(\tilde{u} \tilde{\phi}-u \phi)\|_{L^{q^{\prime}\left(I_{t}, L^{2}\right)}}+C\left\|\left.\tilde{u}\right|^{2 \sigma} \tilde{u}-|u|^{2 \sigma} u\right\|_{L^{q^{\prime}\left(I_{t}, L^{2}\right)}} \\
\leq & C \tau^{\frac{1}{q^{\prime}}}\|\tilde{u}-u\|_{L^{\infty}\left(I_{t}, H^{2}\right)}+C\|\tilde{\phi}-\phi\|_{H^{1}\left(I_{t}\right)}+\tau^{\frac{1}{q^{\prime}}}\|\tilde{u}-u\|_{L^{\infty}\left(I_{t}, L^{2}\right)} .
\end{aligned}
$$

Similarly,

$$
\begin{aligned}
& \left.\|(V(\tilde{u} \tilde{\phi}-u \phi)))_{t}\left\|_{L^{\gamma^{\prime}\left(I_{t}, L^{\rho^{\prime}}\right)}} \leq\right\| V\left(\tilde{\phi}^{\prime}-\phi^{\prime}\right) \tilde{u}\right)\left\|_{L^{\gamma^{\prime}\left(I_{t}, L^{\rho^{\prime}}\right)}}+\right\| V \phi^{\prime}(\tilde{u}-u) \|_{L^{\gamma^{\prime}\left(I_{t}, L^{\rho^{\prime}}\right)}} \\
& \left.+\| V(\tilde{\phi}-\phi) \tilde{u}_{t}\right)\left\|_{L^{\gamma^{\prime}\left(I_{t}, L^{\rho^{\prime}}\right)}}+\right\| V \phi\left(\tilde{u}_{t}-u_{t}\right) \|_{L^{\gamma^{\prime}}\left(I_{t}, L^{\rho^{\prime}}\right)} \\
& \leq C\|\tilde{\phi}-\phi\|_{H^{1}\left(I_{t}\right)}+\tau^{\frac{2-\gamma^{\prime}}{2 \gamma^{\prime}}}\left\|\phi^{\prime}\right\|_{L^{2}}\|\tilde{u}-u\|_{L^{\infty}\left(I_{t}, H^{2}\right)} \\
& +\|V\|_{L^{2 p}}\|\tilde{\phi}-\phi\|_{H^{1}\left(I_{t}\right)}+\|V\|_{L^{2 p}}\left\|\tilde{u}_{t}-u_{t}\right\|_{L^{\gamma^{\prime}\left(I_{t}, L^{2}\right)}} .
\end{aligned}
$$

Notice that the estimates (4.7)-(4.15) hold for $V \in L^{\infty}$. Combining (4.7)-(4.15), using the equivalent norm of $H^{2}$, i.e., $\|\cdot\|_{H^{2}}=\|\cdot\|_{L^{2}}+\|\Delta \cdot\|_{L^{2}}$, we obtain

$$
\begin{aligned}
\|\tilde{u}-u\|_{L^{\infty}\left(I_{t}, H^{2}\right)} \leq & C\|\tilde{u}(t)-u(t)\|_{H^{2}}+C \tau^{\frac{1}{q^{\prime}}}\|\tilde{u}-u\|_{L^{\infty}\left(I_{t}, H^{2}\right)}+C \tau^{\frac{1}{\gamma^{\prime}}}\|\tilde{u}-u\|_{L^{\infty}\left(I_{t}, H^{2}\right)} \\
& +C\|\tilde{\phi}-\phi\|_{H^{1}\left(I_{t}\right)}+\tau^{\frac{2-\gamma^{\prime}}{2 \gamma^{\prime}}}\|\tilde{u}-u\|_{L^{\infty}\left(I_{t}, H^{2}\right)}+\varepsilon\|\tilde{u}-u\|_{L^{\infty}\left(I_{t}, H^{2}\right)} .
\end{aligned}
$$

Therefore, the estimate (4.5) holds by choosing $\tau$ and $\varepsilon$ sufficiently small. Due to $\tilde{u}(0)=u(0)$, we deduce from continuity argument and (4.5) that the mapping $\phi \rightarrow u(\phi)$ is continuous with respect to $\phi \in H^{1}(0, T)$. 
We are now in the position to show Lipschitz continuity of solution $u(\phi)$ with respect to $\phi \in H^{1}(0, T)$. With the estimate (4.5) at hand, the proof is analogue to that of Proposition 4.5 in [11, so we omit it.

Proposition 4.3. Let $N \leq 3, V, \nabla V \in L^{p}+L^{\infty}$ and $V \in L^{2 p}$ for some $p \geq 2$. Assume $0<\sigma<\frac{2}{N-2}$ if $\lambda<0$ or $0<\sigma<\frac{2}{N}$ if $\lambda>0$. Let $\phi \in H^{1}(0, T)$, and $u=u(\phi) \in L^{\infty}\left((0, T), H^{2}\right)$ be the solution of (1.1). Given $\delta_{\phi} \in H^{1}(0, T)$ with $\delta_{\phi}(0)=0$, for every $\varepsilon \in[-1,1]$, let $\tilde{u}=$ $u\left(\phi+\epsilon \delta_{\phi}\right)$ be the solution of (1.1) with control $\phi+\epsilon \delta_{\phi}$ and the same initial data as $u(\phi)$. Then, there exists a constant $C>0$ such that

$$
\|\tilde{u}-u\|_{L^{\infty}\left((0, T), H^{2}\right)} \leq C\|\tilde{\phi}-\phi\|_{H^{1}(0, T)}=C|\varepsilon|\left\|\delta_{\phi}\right\|_{H^{1}(0, T)} .
$$

In other words, the mapping $\phi \mapsto u(\phi)$ is Lipschitz continuous with respect to $\phi$ for each fixed direction $\delta_{\phi}$.

Proof of Theorem 1.2. In view of definition of Gâteaux derivative, let $u=u(\phi), \tilde{u}=u(\tilde{\phi})$ with $\tilde{\phi}=\phi+\varepsilon \delta_{\phi}$, we compute

$$
\mathcal{F}(\tilde{\phi})-\mathcal{F}(\phi)=\mathcal{J}_{1}+\mathcal{J}_{2}+\mathcal{J}_{3}
$$

where

$$
\begin{gathered}
\mathcal{J}_{1}:=\langle\tilde{u}(T), A \tilde{u}(T)\rangle^{2}-\langle u(T), A u(T)\rangle^{2}, \\
\mathcal{J}_{2}:=\gamma_{2} \int_{0}^{T}\left[\left(\tilde{\phi}^{\prime}(t)\right)^{2}-\left(\phi^{\prime}(t)\right)^{2}\right] d t
\end{gathered}
$$

and

$$
\mathcal{J}_{3}:=\gamma_{1} \int_{0}^{T}\left(\tilde{\phi}^{\prime}(t)\right)^{2}\left(\int_{\mathbb{R}^{N}} V(x)|\tilde{\phi}(t, x)|^{2}\right)^{2} d t-\gamma_{1} \int_{0}^{T}\left(\phi^{\prime}(t)\right)^{2}\left(\int_{\mathbb{R}^{N}} V(x)|\phi(t, x)|^{2}\right)^{2} d t .
$$

Because we have obtained Proposition 4.3, $\tilde{u}, u \in L^{\infty}\left((0, T), H^{2}\right) \hookrightarrow L^{\infty}\left((0, T) \times \mathbb{R}^{N}\right)$, ones can prove along the lines of Theorem 4.6 in [1], so we omit it.

\section{References}

[1] V. Bulatov, B. E. Vugmeister, H. Rabitz, Nonadiabatic control of Bose-Einstein condensation in optical traps, Phys. Rev. A, 60(1999) 4875-4881.

[2] U. Hohenester, P. K. Rekdal, A. Borzi, J. Schmiedmayer, Optimal quantum control of Bose Einstein condensates in magnetic microtraps, Phys. Rev. A, 75(2007) 023602-023613.

[3] M. Holthaus, Toward coherent control of Bose-Einstein condensate in a double well, Phys. Rev. A, 64(2001) 011601-011608.

[4] J.-M. Coron, Control and Nonlinearity. Mathematical Surveys and Monographs, vol. 136, American Mathematical Society, 2007.

[5] H. Fattorini, Infinite dimensional optimization and control theory, Cambridge University Press, 1999. 
[6] J.L. Lions, Optimal control of systems governed by partial differential equations, Springer Verlag, 1971.

[7] K. Ito, K. Kunisch, Optimal bilinear control of an abstract Schrödinger equation, SIAM J. Control Optim., 46(2007) 274-287.

[8] L. Baudouin, O. Kavian, J.P. Puel, Regularity for a Schrödinger equation with singular potentials and application to bilinear optimal control, J. Diff. Equ., 216(2005) 188-222.

[9] L. Baudouin, J. Salomon, Constructive solution of a bilinear optimal control problem for a Schrödinger equation, Systems Control Lett., 57(2008) 454-464.

[10] B. Yildiz, O. Kilicoglu, G. Yagubov, Optimal control problem for nonstationary Schrödinger equation, Num. Methods Partial Diff. Equ., 25(2009) 1195-1203.

[11] M. Hintermüller, D. Marahrens, P.A. Markowich, C. Sparber, Optimal bilinear control of GrossPitaevskii equations, Arxiv preprint arXiv:1202.2306.

[12] T. Cazenave, Semilinear Schrödinger equations, Courant Lecture Notes in Mathematics vol. 10, New York University, Courant Institute of Mathematical Sciences, New York; American Mathematical Society, Providence, RI, 2003. 